\title{
Combined Effects of Amino Acids in Garlic and Buna-Shimeji (Hypsizygus marmoreus) on Suppression of $\mathrm{CCl}_{4}$-Induced Hepatic Injury in Rats
}

\author{
Yusuke Yamaguchi ${ }^{1}{ }^{(0}$, Yushi Hirata ${ }^{1}$, Takeshi Saito ${ }^{2}$ and Hitomi Kumagai ${ }^{1, *}$ \\ 1 Department of Chemistry and Life Science, Nihon University, 1866 Kameino, Fujisawa-shi 252-0880, Japan; \\ yamaguchi.yusuke@nihon-u.ac.jp (Y.Y.); ihsuy0513@gmail.com (Y.H.) \\ 2 ACERA Co., Ltd., 156 Nishitakahashi-machi, Kofu-shi 400-0826, Japan; t-saito@acera-jp.com \\ * Correspondence: kumagai.hitomi@nihon-u.ac.jp; Tel.: +81-466-3946
}

check for updates

Citation: Yamaguchi, Y.; Hirata, Y.; Saito, T.; Kumagai, H. Combined Effects of Amino Acids in Garlic and Buna-Shimeji (Hypsizygus marmoreus) on Suppression of $\mathrm{CCl}_{4}$-Induced Hepatic Injury in Rats. Foods 2021, 10, 1491. https://doi.org/10.3390/ foods10071491

Academic Editors: Chibuike

C. Udenigwe and Rotimi Aluko

Received: 17 May 2021

Accepted: 22 June 2021

Published: 27 June 2021

Publisher's Note: MDPI stays neutral with regard to jurisdictional claims in published maps and institutional affiliations.

Copyright: (C) 2021 by the authors Licensee MDPI, Basel, Switzerland. This article is an open access article distributed under the terms and conditions of the Creative Commons Attribution (CC BY) license (https:// creativecommons.org/licenses/by/ $4.0 /$ )

\begin{abstract}
The combination of the garlic-derived amino acid, S-allyl-L-cysteine sulfoxide (ACSO), and ornithine or arginine on $\mathrm{CCl}_{4}$-induced hepatic injury was examined. After investigating the effectiveness of the mixture of ACSO and ornithine or arginine in preventing hepatic injury in vivo, an extract rich in ACSO and ornithine was prepared by converting arginine in garlic to ornithine by arginase from Hypsizygus marmoreus (buna-shimeji), after screening the productivity of ornithine among 12 kinds of mushrooms. Co-administration of ACSO with ornithine or arginine suppressed the increase in aspartate transaminase, alanine transaminase, and thiobarbituric acid reactive substance, and the decrease in glutathione $S$-transferase and cytochrome p450 $2 \mathrm{E} 1$ activities after $\mathrm{CCl}_{4}$ injection more effectively than a single administration of ACSO. All extracts prepared from garlic and bunashimeji with low and high contents of ACSO and arginine or ornithine significantly suppressed $\mathrm{CCl}_{4}$-induced hepatic injury in rats. Considering that ACSO is tasteless, odourless, and enhances taste, and ornithine has a flat or sweet taste and masks bitterness, the extract rich in ACSO and ornithine from garlic and buna-shimeji could be considered a potential antioxidant food material that can be added to many kinds of food to prevent hepatic injury.
\end{abstract}

Keywords: garlic; mushroom; Hypsizygus marmoreus; buna-shimeji; ACSO; alliin; arginine; ornithine; hepatic injury

\section{Introduction}

Oxidative stress is highly related to diseases such as cancer, vascular diseases, and Alzheimer's disease [1-3]. Excessive amounts of oxidative stress-inducing agents generated in vivo, such as hydroxyl radicals, superoxide anion radicals, and hydrogen peroxide, should be quenched with antioxidative and detoxifying compounds for the prevention of such diseases [4-6]. Food and its components, such as phytochemicals [5,7], vitamin E [8], and others $[9,10]$, which play a role in the defence against oxidative stress, have attracted significant attention. These food materials could be effective in preventing oxidative stress-induced diseases.

Garlic has been widely used as a medicinal herb and is a promising food material against oxidative stress [11]. The major medicinal components in garlic are diallyl disulphide (DADS) and diallyl trisulfide (DATS) [12-17], which increase antioxidant activity [18-22]. When garlic is crushed or sliced, these sulphides, which are responsible for the characteristic garlic odour, are produced from an amino acid, S-allyl-L-cysteine sulfoxide (ACSO; also known as alliin), by cysteine-S-conjugate $\beta$-lyase (C-S lyase), followed by spontaneous reactions of sulfenic acid [23]. Although these sulphides show potent antioxidant effects, it is difficult to use them as food materials because they are volatile, hydrophobic, and have a strong odour. In contrast, ACSO, a dominant amino acid in raw garlic, is non-volatile, hydrophilic, odourless, tasteless, and a taste enhancer [24]. It can 
be used in many kinds of food without impairing palatability. We have already shown that ACSO has antioxidant activity, induces antioxidative and detoxifying enzymes, and suppresses hepatic injury in rats [25]. Another dominant amino acid in garlic is arginine, which is hydrolysed to ornithine by arginase. Although there is little scientific evidence that ornithine alleviates hepatic injury despite the common assumption of its function in Japan, ornithine aspartate, a stable salt, reportedly shows hepatoprotective activity against thioacetamide-induced hepatopathy [26].

Some mushrooms have highly active arginase that catalyses the hydrolysis of arginine to ornithine and urea, accumulating a considerable amount of urea in the fruit bodies to regulate osmotic pressure [27]. Thus, an extract rich in ACSO and ornithine could be prepared by mixing garlic extracts with inactivated C-S lyase and mushroom extracts with arginase. In addition, as compounds in the urea cycle that convert toxic ammonia to urea, arginine and ornithine may protect hepatocytes via a different mechanism from ACSO. Therefore, the concomitant use of ACSO and arginine or ornithine may more effectively prevent hepatic injury induced by toxic agents, such as $\mathrm{CCl}_{4}$ and ammonia, than the single use of ACSO.

In this study, the protective effect of co-administration of synthesised ACSO with ornithine or arginine against $\mathrm{CCl}_{4}$-induced hepatic injury was investigated. We then screened mushrooms that effectively converted arginine to ornithine. After selecting the mushroom, the garlic extract with low or high ACSO content was mixed with the mushroom extract containing arginase to convert arginine to ornithine. In addition, a garlic extract with a low or high ACSO content not mixed with the mushroom extract was prepared to examine the effect of arginine. The antioxidant effects of these extracts were evaluated in terms of the suppression of $\mathrm{CCl}_{4}$-induced hepatic injury, which has been well studied and is suitable for the evaluation of antioxidative and detoxifying effects of food materials [28-31]. This study proposes a novel food combination to present a potent antioxidative effect.

\section{Materials and Methods}

\subsection{Materials}

ACSO was chemically synthesised according to previous reports [25,32]. L-Arginine was purchased from Daesang Co., Ltd. (Seoul, Korea). L-Ornithine was purchased from Kyowa Hakko Bio Co., Ltd. (Tokyo, Japan). Other chemical reagents used in this study were purchased from FUJIFILM Wako Pure Chemical Corporation (Osaka, Japan), Kanto Chemical Co., Inc. (Tokyo, Japan), Oriental Yeast Co., Ltd. (Tokyo, Japan), Cosmobio Co., Ltd. (Tokyo, Japan), Nacalai Tesque, Inc. (Kyoto, Japan), and Roche Diagnostics GmbH Co., Ltd. (Mannheim, Germany).

\subsection{Screening of Mushrooms with High Ornithine Production}

Twelve kinds of mushrooms, namely, Hypsizygus marmoreus (buna-shimeji), Pleurotus eryngii, Agaricus blazei, Flammulina velutipes, Pleurotus eryngii var. tuoliensis, Pleurotus ostreatus, Hericium erinaceus, Pleurotus cornucopiae, Agaricus bisporus, Lyophyllum decastes, Lentinula edodes, and Grifola frondosa, were purchased from a local market. Frozen mushrooms were homogenised in deionised water of the same weight using a household juicer. The homogenate was centrifuged at $3000 \times \mathrm{g}$ for $10 \mathrm{~min}$, and the supernatant was collected. The supernatant $(20 \mathrm{~mL})$ was mixed with $0.4 \mathrm{~g}$ of arginine and adjusted to $\mathrm{pH} 10$ using $1 \mathrm{M} \mathrm{NaOH}$. The mixture was stirred at $50{ }^{\circ} \mathrm{C}$ for $8 \mathrm{~h}$. The ornithine, arginine, urea, and ammonia concentrations in the resultant supernatant were measured using an amino acid analyser (JLC-500/V, JEOL Ltd. Tokyo, Japan).

\subsection{Preparation of Garlic Extract, Garlic Plus Buna-Shimeji Extract, Heated-Garlic Extract, and} Heated-Garlic Plus Buna-Shimeji Extract

Peeled garlic obtained from a local market was chopped with a household food chopper (MK-K78, Panasonic, Osaka, Japan) and homogenised using a homogeniser (Ace 
homogenizer, Nihonseikikaisha Ltd., Tokyo, Japan). The homogenate was stirred in 75\% ethanol, and the solution was centrifuged at $3000 \times g$. The supernatant was lyophilised, and the dried residue was designated as the garlic extract $(\mathrm{Ge})$.

The pileus of buna-shimeji obtained from a local market was frozen. Frozen pileus and Ge were mixed and homogenised. The $\mathrm{pH}$ of the homogenate was adjusted to 9.5 with $1 \mathrm{M}$ $\mathrm{NaOH}$. The homogenate was kept for $16 \mathrm{~h}$ at $40{ }^{\circ} \mathrm{C}$ to convert arginine to ornithine. After the conversion was confirmed using an amino acid analyser, $75 \%$ ethanol was added to the homogenate and stirred for an hour. The solution was centrifuged at $3000 \times g$, and the supernatant was concentrated under vacuum to remove ethanol, followed by neutralisation using citric acid. The dried residue after lyophilisation of the neutralised supernatant was designated as garlic plus buna-shimeji extract (GBe).

Peeled garlic obtained from the local market was heated in hot water at $80^{\circ} \mathrm{C}$ for $30 \mathrm{~min}$. The heated garlic was cooled to room temperature (ca. $25^{\circ} \mathrm{C}$ ), chopped, and homogenised. The homogenate was stirred in $75 \%$ ethanol, and the solution was centrifuged at $3000 \times g$. The supernatant was concentrated under vacuum to remove ethanol and lyophilised. The dried residue was designated as heated-garlic extract (HGe).

Heated-garlic plus buna-shimeji extract (HGBe) was prepared by replacing Ge with HGe in the same way used for the preparation of GBe.

The ACSO content was determined using high performance liquid chromatography (HPLC) after derivatisation with o-phthalaldehyde. The contents of L-arginine, L-ornithine, and other amino acids were determined using an amino acid analyser.

\subsection{Animals}

Five-week-old male Sprague Dawley rats were purchased from Japan SLC, Inc. (Tokyo, Japan). Experiments on the animals were performed in accordance with the Guidelines for Animal Experiments of the College of Bioresource Sciences, Nihon University (approval code: AP13B010). The rats were housed in individual, stainless-steel, wire cages with free access to food (CE-2, Clea Japan, Tokyo, Japan) and water during a seven-day acclimation period before the experiments. The feeding facility was maintained at approximately $21^{\circ} \mathrm{C}$ with a $12 \mathrm{~h}$ light-dark cycle.

\subsection{Effect of Co-Administration of ACSO with Arginine or Ornithine on Suppression of Hepatic Injury Induced by $\mathrm{CCl}_{4}$}

The rats were randomly divided into eight groups of six rats each. ACSO was dissolved in distilled water and orally administered via gavage at a dosage of $100 \mu \mathrm{mol} / \mathrm{mL} /$ day for five consecutive days. A mixture of $50 \mu \mathrm{mol}$ of ACSO and $50 \mu \mathrm{mol}$ of L-ornithine or Larginine in $1 \mathrm{~mL}$ of distilled water was orally administered via gavage for five consecutive days in the $\mathrm{ACSO}+$ arginine (ACSO + Arg) or ACSO + ornithine (ACSO + Orn) group, respectively. The distilled water $(\mathrm{DW})$ group received only $1 \mathrm{~mL}$ of distilled water per day via gavage for five consecutive days. $\mathrm{CCl}_{4}$ was intraperitoneally administered at a dosage of $0.5 \mathrm{~mL} / \mathrm{kg}$ body weight after the 5 th oral administration of samples in the $\mathrm{CCl}_{4}$ groups. The rats were then subjected to fasting for $18 \mathrm{~h}$, and their body weight was measured. The blood was collected, treated with sodium citrate, and centrifuged at $1500 \times g$ for $15 \mathrm{~min}$ at $4{ }^{\circ} \mathrm{C}$ to obtain the serum for further experiments. The liver was excised, and its weight was measured. The microsomal and cytosolic fractions were prepared by centrifugation, as described previously [33]. These fractions were used in further experiments.

\subsection{Effect of GBe on Suppression of Hepatic Injury Induced by $\mathrm{CCl}_{4}$}

The rats were randomly divided into 10 groups of six rats each. Briefly, $10 \%$ of Ge, GBe, $\mathrm{HGe}$, and HGBe were dissolved in distilled water, and the solution was orally administered at a dosage of $1 \mathrm{~mL} /$ day via gavage for five consecutive days to the corresponding group. The DW group received only $1 \mathrm{~mL}$ of distilled water per day via gavage for five consecutive days. $\mathrm{CCl}_{4}$ was intraperitoneally administered at a dosage of $0.5 \mathrm{~mL} / \mathrm{kg}$ body weight after the 5 th oral administration of samples in the $\mathrm{CCl}_{4}$ groups. The remaining procedure was the same as that described in Section 2.5. 


\subsection{Determination of Aspartate Transaminase and Alanine Transaminase Levels}

The serum obtained was used to determine aspartate transaminase (AST) and alanine transaminase (ALT) levels using an enzymatic method with an automatic analyser, Spotchem EZ SP-4430 (Liver-2, Arkray, Inc., Kyoto, Japan).

\subsection{Measurement of CYP2E1 Activity}

The activity of cytochrome p450 2E1 (CYP2E1) was determined according to a previously described method [34]. Briefly, the microsome fraction of the liver was incubated at $37^{\circ} \mathrm{C}$ for $30 \mathrm{~min}$ in a potassium phosphate buffer containing nicotinamide adenine dinucleotide phosphate (NADP), D-glucose-6-phosphate, magnesium chloride, glucose-6phosphate dehydrogenase, sodium citrate, and $p$-nitrophenol. Then, trichloroacetic acid was added to the solution and cooled in an ice bath. The mixture was centrifuged, and the supernatant was collected. The supernatant was mixed with $2 \mathrm{M} \mathrm{NaOH}$, and the absorbance at $535 \mathrm{~nm}$ was measured to determine the amount of $p$-nitrocatechol produced by the oxidation of $p$-nitrophenol by CYP2E1. The protein concentration of the microsome fraction of the liver was determined using the bicinchoninic-acid assay.

\subsection{Measurement of Lipid Peroxide}

The lipid peroxide content in the liver was determined using a thiobarbituric acidreactive substance (TBARS) assay, according to a previous report [25]. Briefly, the liver homogenate was mixed with $1 \%$ phosphoric acid and $0.67 \%$ thiobarbituric acid (TBA) solution. The mixture was incubated at $95^{\circ} \mathrm{C}$ for $45 \mathrm{~min}$, and then aldehyde-TBA adduct was extracted using $n$-butanol. The absorbance of aldehyde-TBA adduct at $535 \mathrm{~nm}$ was measured. Malondialdehyde (MDA) was used as a standard, and the TBARS value was expressed as MDA equivalent.

\subsection{Measurement of Glutathione S-Transferase Activity}

The activity of glutathione $S$-transferase (GST) in the cytosol fraction of the liver was assayed spectrophotometrically according to a previous report [25]. Briefly, the cytosol fraction from the liver and 1-chloro-2,4-dinitrobenzene (CDNB) were added to a solution containing $30 \mathrm{mM}$ reduced glutathione (GSH) in $0.1 \mathrm{M}$ potassium phosphate buffer at $\mathrm{pH} 7.4$ at $25^{\circ} \mathrm{C}$. The increase in the absorbance at $340 \mathrm{~nm}$ attributed to $S-2,4-$ dinitrophenylglutathione was measured. The activity of GST was expressed as the amount of S-2,4-dinitrophenylglutathione produced per minute per milligram of cytosol protein. The protein concentration of the cytosol fraction of the liver was determined using the bicinchoninic-acid assay.

\subsection{Statistical Analysis}

All data are expressed as mean \pm standard deviation (SD), and the significance of the differences between groups was evaluated using a one-way analysis of variance followed by Tukey-Kramer's test.

\section{Results}

\subsection{Effect of Co-Administration of Arginine or Ornithine with ACSO on $\mathrm{CCl}_{4}$-Induced} Hepatic Injury

The liver weight, AST and ALT activities, and TBARS increased, whereas CYP2E1 and GST activities decreased in the DW group after the intraperitoneal injection of $\mathrm{CCl}_{4}$, which are typical changes observed in rats with $\mathrm{CCl}_{4}$-induced hepatic injury (Figures 1 and 2). These changes induced by $\mathrm{CCl}_{4}$ were significantly suppressed in the ACSO, ACSO $+\mathrm{Arg}$, and ACSO + Orn groups, even after $\mathrm{CCl}_{4}$ injection $(p<0.01)$. AST and ALT activities and TBARS in the ACSO + Arg and ACSO + Orn groups treated with $\mathrm{CCl}_{4}$ were lower than those in the ACSO group treated with $\mathrm{CCl}_{4}$. CYP2E1 and GST activities in the ACSO + Arg and ACSO + Orn groups treated with $\mathrm{CCl}_{4}$ were higher than those in the ACSO group treated with $\mathrm{CCl}_{4}$. In particular, AST activity of the $\mathrm{ACSO}+$ Orn group treated with $\mathrm{CCl}_{4}$ 
was significantly lower than that of the ACSO group treated with $\mathrm{CCl}_{4}(p<0.01)$. ALT activity of the ACSO $+\mathrm{Arg}$ and $\mathrm{ACSO}+$ Orn groups treated with $\mathrm{CCl}_{4}$ was significantly lower than that of the ACSO group treated with $\mathrm{CCl}_{4}(p<0.01)$. In the absence of $\mathrm{CCl}_{4}$ administration, GST activity in the ACSO, ACSO + Arg, and ACSO + Orn groups was significantly higher than that in the DW group $(p<0.05)$.

(a)

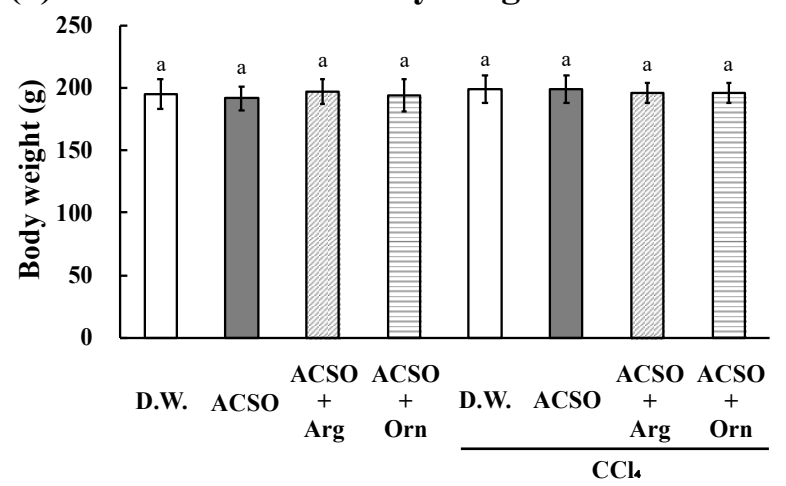

(b)

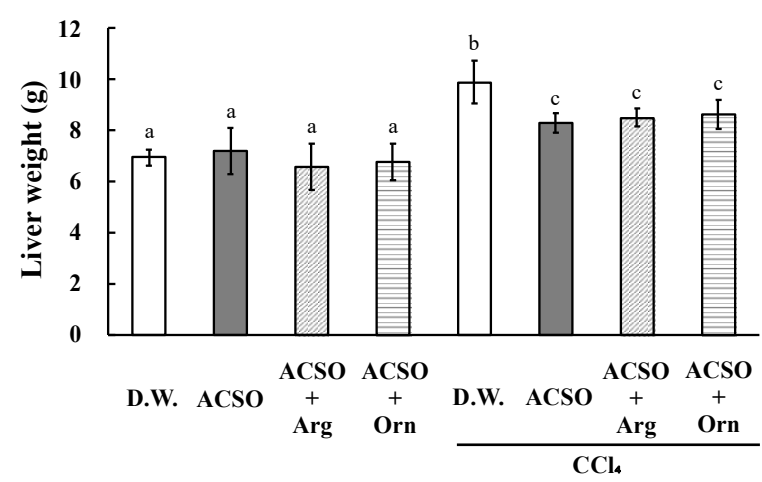

Figure 1. Effect of co-administration of arginine or ornithine with ACSO on body weight (a), and the liver weight (b) of rats with $\mathrm{CCl}_{4}$-induced hepatic injury. Six rats in each group received a sample (ACSO, ACSO + Arg, or ACSO + Orn) orally for 5 consecutive days. $\mathrm{CCl}_{4}$ was intraperitoneally injected, then body weight and liver weight were measured. Co-administration of arginine or ornithine with ACSO suppressed the increase in the liver weight. Each value represents the mean \pm standard deviation (SD). The different letters with bars in the figures indicate a significant difference between the groups $(p<0.01)$. The same letters with bars indicate no significant difference between the groups $(p<0.01)$. 
(a)

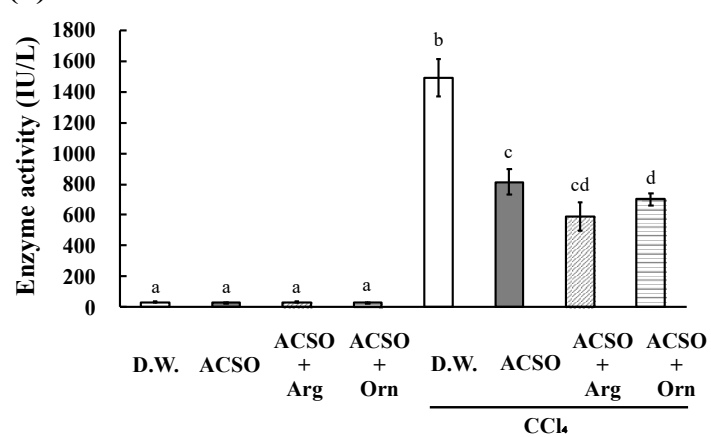

(c)

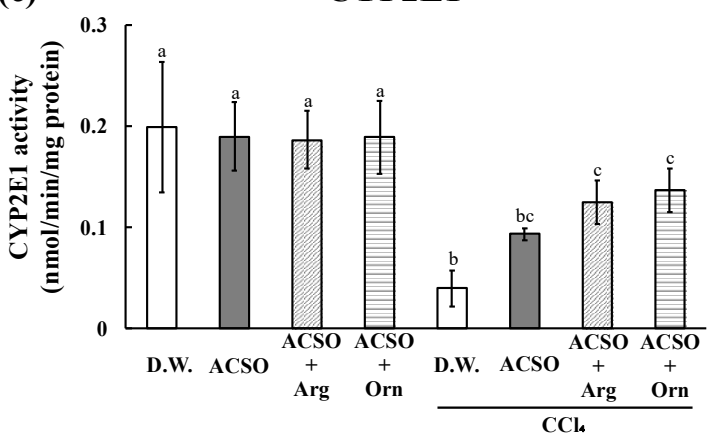

(b)

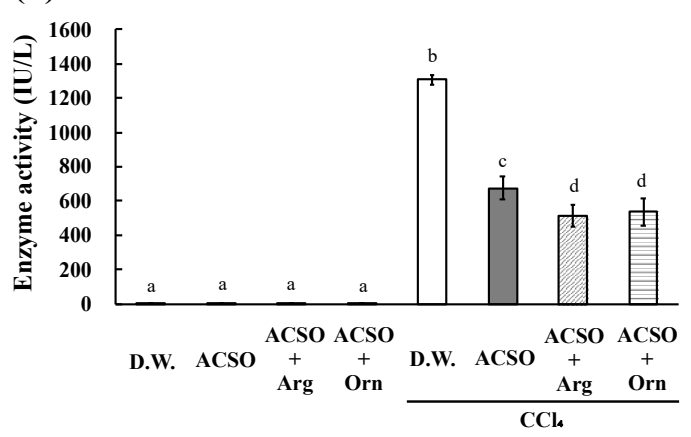

(d)

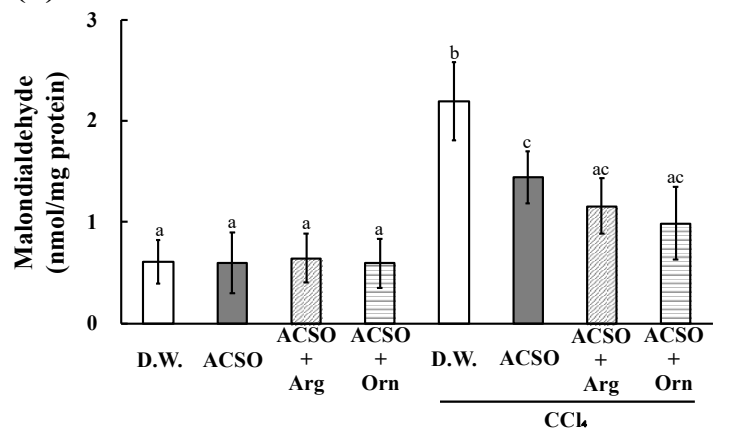

(e)

GST

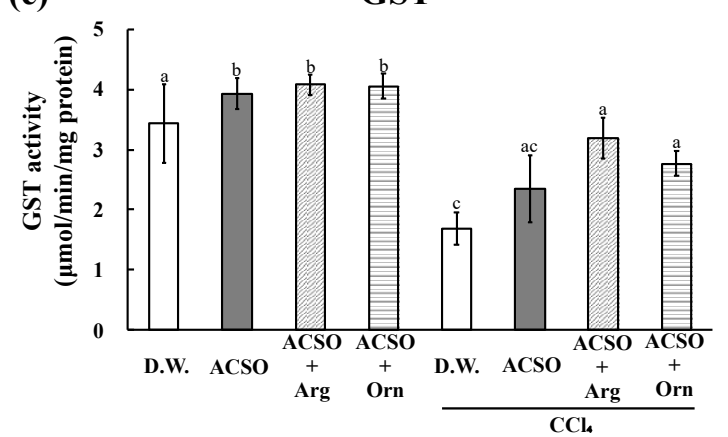

Figure 2. Effect of co-administration of arginine or ornithine with ACSO on aspartate transaminase (AST) activity (a), alanine transaminase (ALT) activity (b), cytochrome p450 2E1 (CYP2E1) activity (c), amount of thiobarbituric acid reactive substances (TBARS) (d), and glutathione-S-transferase (GST) activity (e) in rats with $\mathrm{CCl}_{4}$-induced hepatic injury. Six rats in each group received a sample (ACSO, ACSO $+\mathrm{Arg}$, or ACSO + Orn) orally for 5 consecutive days. $\mathrm{CCl}_{4}$ was intraperitoneally injected, and the blood and the liver were collected to assess the degree of hepatic injury. Co-administration of arginine or ornithine with ACSO suppressed the increases in AST and ALT levels more than a single administration of ACSO. Each value represents the mean $\pm \mathrm{SD}$. The different letters with bars in figures $(\mathbf{a}-\mathbf{d})$ indicate a significant difference between the groups $(p<0.01)$. The different letters with bars in figure $(\mathbf{e})$ indicate a significant difference between the groups $(p<0.05)$. The same letters indicate no significant difference between the groups in figures (a-d) at $p<0.01$ and in figure (e) at $p<0.05$.

\subsection{Ornithine Production by Mushrooms}

The ornithine, arginine, urea, and ammonia contents in the mushroom extract after arginine addition are shown in Figure 3. H. marmoreus, P. eryngii, A.blazei, F. velutipes, $P$. eryngii var. tuoliensis, and $P$. ostreatus showed high ornithine concentration $(86,93,95,91$, 105 , and $116, \mu \mathrm{mol} / \mathrm{mL}$, respectively) in the extract. The ornithine concentration in these solutions before arginine addition was approximately $4 \mu \mathrm{mol} / \mathrm{mL}$. Urea was not detected 
in the H. marmoreus extract. As ammonia can be removed by lyophilisation during sample preparation, we decided to use H. marmoreus to convert arginine in garlic.

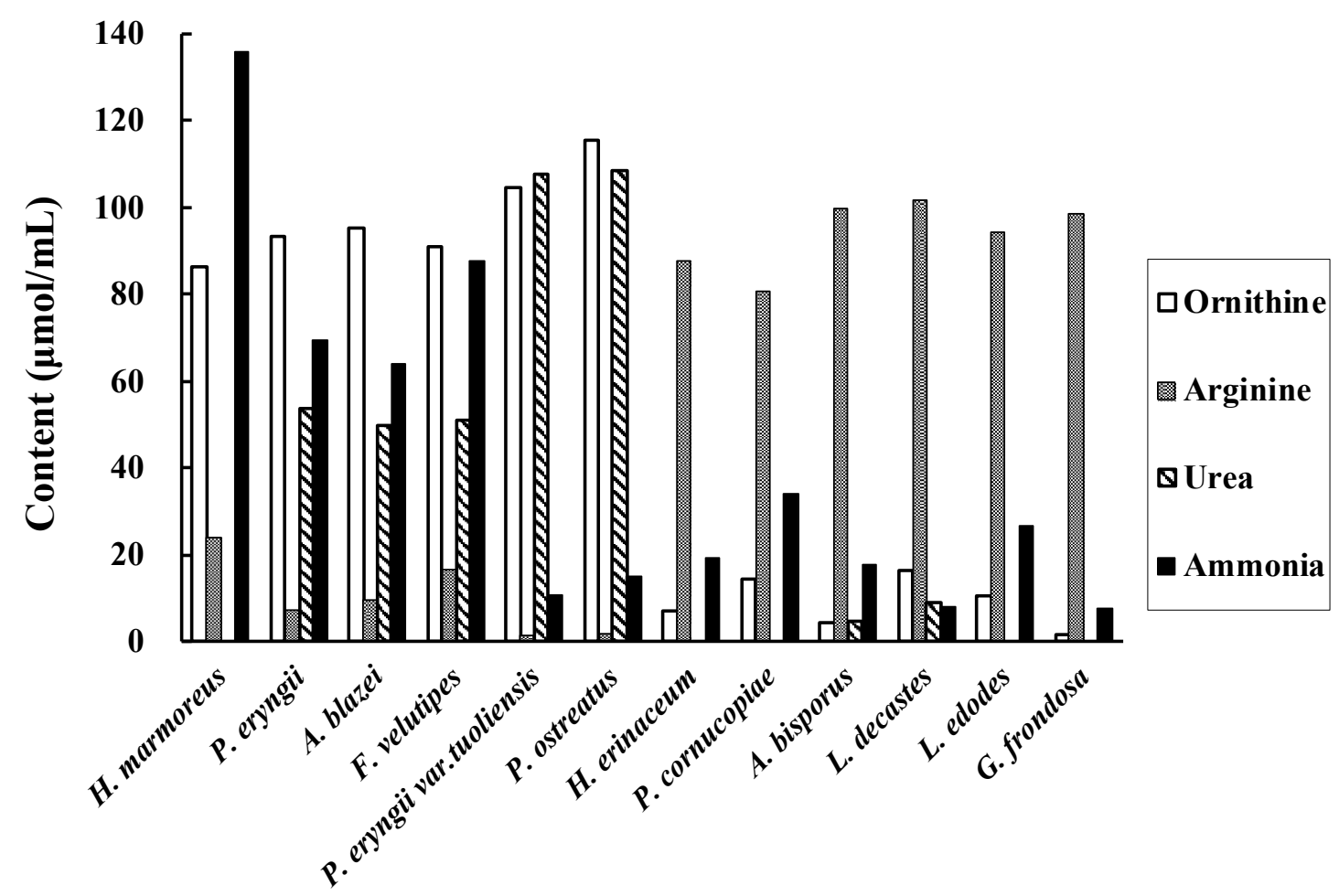

Figure 3. Ornithine, arginine, urea, and ammonia concentrations after arginine addition in the extracts of 12 kinds of mushrooms.

3.3. Contents of Amino Acids including ACSO, Arginine, and Ornithine in Ge, GBe, $H G e$, and $\mathrm{HGBe}$

Among the three compounds, ACSO, arginine, and ornithine, Ge predominantly contained arginine, while GBe predominantly contained ornithine (Table 1). On the other hand, HGe primarily contained both ACSO and arginine, while HGBe primarily contained both ACSO and ornithine. The contents of all the other amino acids were lower than $1 \%$ in Ge, Gbe, HGe, and HGBe (Table S1). The contents of aspartic acid, cystine, methionine, and tyrosine, reported to suppress $\mathrm{CCl}_{4}$-induced hepatic injury [35], were much lower than those of ACSO, arginine, and ornithine in the extracts.

Table 1. Contents of ACSO, arginine, and ornithine in garlic and bunashimeji extracts.

\begin{tabular}{cccc}
\hline Group & ACSO (\%) & Arginine (\%) & Ornithine (\%) \\
\hline Ge & 0.08 & 3.19 & 0.02 \\
GBe & 0.13 & 0.04 & 1.69 \\
HGe & 1.45 & 3.16 & 0.03 \\
HGBe & 1.16 & 0.01 & 1.97 \\
\hline
\end{tabular}

\subsection{Effect of $\mathrm{Ge}, \mathrm{Gbe}, \mathrm{HGe}$, and $\mathrm{HGBe}$ on $\mathrm{CCl}_{4}$-Induced Hepatic Injury}

The elevation in liver weight, AST, ALT, and TBARS induced by $\mathrm{CCl}_{4}$ injection was significantly suppressed in the Ge, GBe, HGe, and HGBe groups ( $p<0.05$, Figures 4 and 5). The decrease in CYP2E1 and GST activities induced by $\mathrm{CCl}_{4}$ injection was also suppressed in the Ge, GBe, HGe, and HGBe groups $(p<0.05)$. GST activities in the Ge, GBe, HGe, and HGBe groups were higher than those in the DW group in the absence of $\mathrm{CCl}_{4}$ administration, but the difference was not considered significant. 

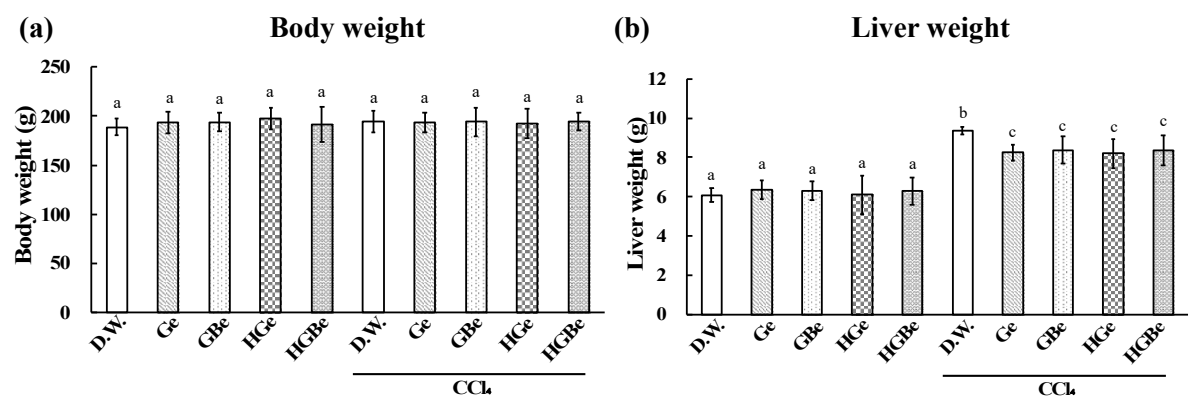

Figure 4. Effect of $\mathrm{Ge}, \mathrm{GBe}, \mathrm{HGe}$, and $\mathrm{HGBe}$ on body weight (a) and liver weight (b) of rats with $\mathrm{CCl}_{4}$-induced hepatic injury. Six rats in each group received a sample (Ge, GBe, $\mathrm{HGe}$, or $\mathrm{HGBe}$ ) orally for 5 consecutive days. $\mathrm{CCl}_{4}$ was intraperitoneally injected, then body weight and the liver weight were measured. The extract of garlic and garlic with buna-shimeji suppressed the increase in liver weight. Each value represents the mean $\pm \mathrm{SD}$. The different letters with bars in the figures indicate a significant difference between the groups $(p<0.05)$. The same letters with bars indicate no significant difference between the groups $(p<0.05)$.
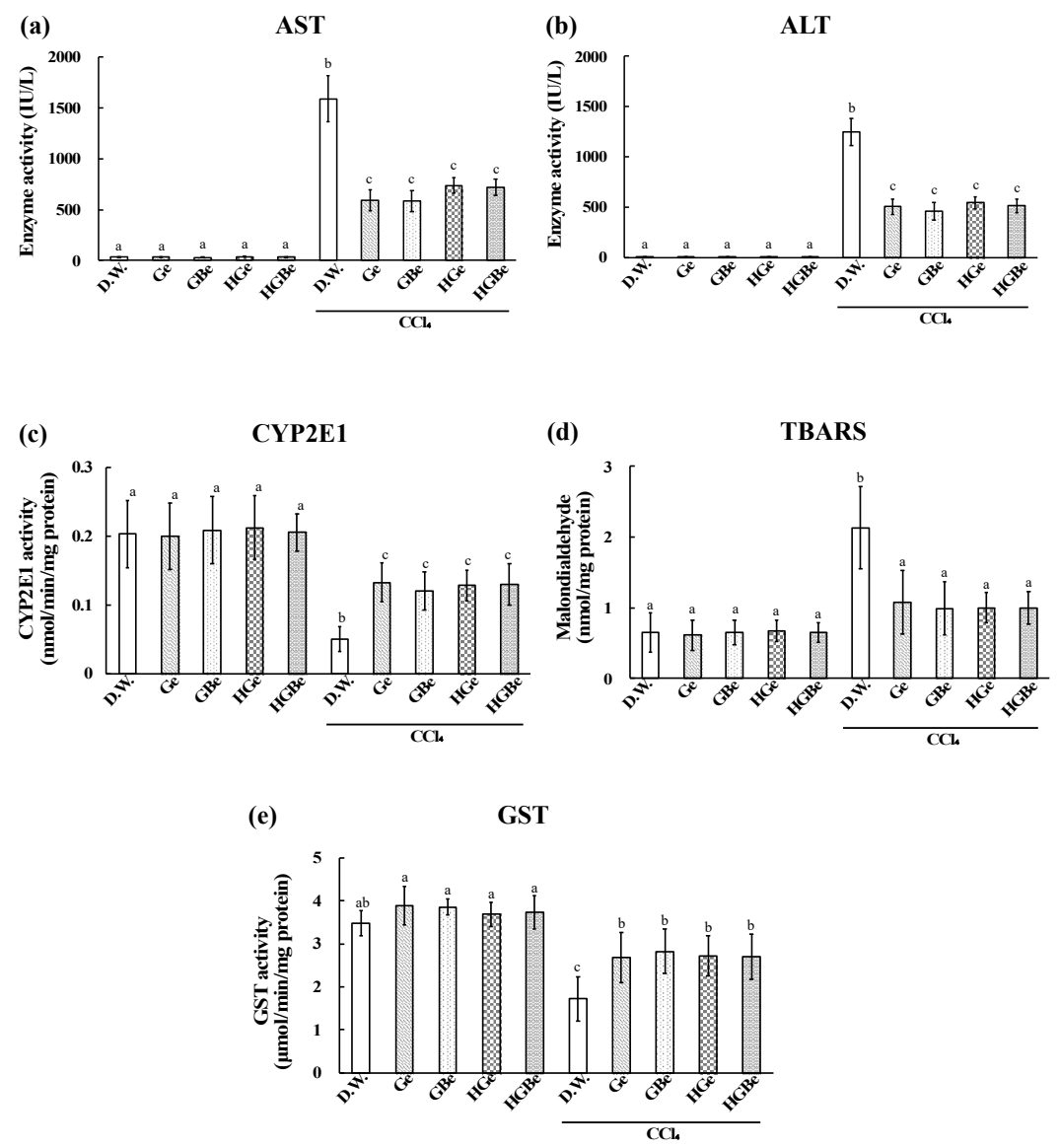

Figure 5. Effect of Ge, GBe, HGe, and HGBe on aspartate transaminase (AST) activity (a), alanine transaminase (ALT) activity (b), cytochrome p450 2E1 (CYP2E1) activity (c), amount of thiobarbituric acid reactive substances (TBARS) (d), and glutathione-S-transferase (GST) activity (e) in rats with $\mathrm{CCl}_{4}$-induced hepatic injury. Six rats in each group received an extract (Ge, GBe, $\mathrm{HGe}$, or HGBe) orally for 5 consecutive days. $\mathrm{CCl}_{4}$ was intraperitoneally injected, and the blood and the liver were collected to assess the degree of hepatic injury. The extract of garlic and garlic with buna-shimeji suppressed the increases in AST, ALT, and TBRAS levels and the decreases in CYP2E1 and GST levels. Each value represents the mean \pm SD. The different letters with bars in figure $(\mathbf{d})$ indicate a significant difference between the groups $(p<0.01)$. The different letters with bars in the figures $(\mathbf{a}-\mathbf{c}, \mathbf{e})$ indicate a significant difference between the groups $(p<0.05)$. The same letters with bars indicate no significant difference between the groups in figure (d) at $p<0.01$ and in figures (a-c,e) at $p<0.05$. 


\section{Discussion}

Garlic is known to have various physiological functions, and sulphides such as DADS and DATS are considered to be the major active components of garlic. In our previous study, we showed that ACSO, an odour precursor, also has various functions, such as prevention of $\mathrm{CCl}_{4}$-induced hepatic injury, inhibition of platelet aggregation, and suppression of the increase in blood ethanol levels [25,36,37].

After intraperitoneal injection, $\mathrm{CCl}_{4}$ is reduced by cytochrome P450 2E1 (CYP2E1) to the $\mathrm{CCl}_{3}$ radical in the liver [38,39], which forms covalent bonds with proteins, nucleic acids, and lipids, thus impairing their functions [40,41]. Moreover, $\mathrm{CCl}_{3}$ radicals oxidise lipids to produce lipid peroxide, in addition to destroying the lipid bilayer of cell membranes, resulting in leakage of the contents of the liver cells, such as AST and ALT, into the blood [42]. Lipid peroxide is reduced by glutathione (GSH) or conjugated with GSH by GST and excreted. Thus, the preventive effect on $\mathrm{CCl}_{4}$-induced hepatic injury was evaluated based on AST and ALT activities in the blood, CYP2E1 and GST activities, and lipid peroxide value in the liver after oral administration of samples following intraperitoneal injection of $\mathrm{CCl}_{4}$. The injection of $\mathrm{CCl}_{4}$ significantly increased AST and ALT activities and TBARS values but decreased CYP2E1 and GST activities. However, co-administration of arginine/ornithine with ACSO and a single administration of ACSO effectively suppressed hepatic injury (Figure 2).

One of the mechanisms for preventing $\mathrm{CCl}_{4}$-induced hepatic injury by $\mathrm{ACSO}$ is the promotion of nuclear translocation of NF-E2-related factor 2 (Nrf2) to induce antioxidative and detoxifying enzymes [25]. Arginine suppresses oxidative stress by promoting Nrf2 translocation to the nucleus [43]. In contrast, ornithine aspartate lowers the blood ammonia concentration [44], which is considered to be one of the reasons for its hepatoprotective activity [26]. In addition, the administration of ornithine aspartate suppressed the decrease in liver tissue GSH level caused by thioacetamide-induced hepatopathy [26]. Ornithine may also function as a suppressor of oxidative stress caused by $\mathrm{CCl}_{4}$, probably owing to the biosynthesis of GSH via glutamate [45]. As a compound in the urea cycle that is converted to ornithine, arginine may have worked in a way similar to ornithine. Although the dose of ornithine aspartate previously [26] was approximately $200 \mu \mathrm{mol}$, we administered $50 \mu \mathrm{mol}$ of ornithine/arginine together with $50 \mu \mathrm{mol}$ of ACSO in $1 \mathrm{~mL}$ of distilled water to rats for five consecutive days before intraperitoneal injection of $\mathrm{CCl}_{4}$ and compared the effect with that of $100 \mu \mathrm{mol}$ of ACSO. Although the dose of ornithine/arginine in this study was lower than that of ornithine aspartate $(200 \mu \mathrm{mol})$ in the literature [26], and the dose of ACSO in the ACSO + Orn and ACSO + Arg groups was lower than that in the ACSO group, the co-administration of $50 \mu \mathrm{mol}$ of ornithine/arginine with $50 \mu \mathrm{mol}$ of ACSO suppressed the increase in AST and ALT activities more potently than the administration of $100 \mu \mathrm{mol}$ ACSO. This result indicates that the combination of ornithine/arginine and ACSO showed a synergistic effect. One of the reasons for this synergistic effect might be the nuclear translocation of Nrf2 by ACSO and arginine and the elimination of toxic ammonia by ornithine/arginine.

Some mushrooms are considered to have arginase, which converts arginine to ornithine and urea, and urease, which hydrolyses urea to ammonia and carbon dioxide. Therefore, to examine the activities of arginase and urease in mushrooms, urea, ammonia, ornithine, and arginine contents in mushroom extracts were measured after the addition of arginine and incubation at $50{ }^{\circ} \mathrm{C}$ for $8 \mathrm{~h}$. Among the mushrooms examined, H. marmoreus, P. eryngii, A. blazei, F. velutipes, P. eryngii var. tuoliensis, and P. ostreatus showed high ornithine levels, indicating high arginase activity. H. marmoreus contained no urea; nonetheless, it contained the largest amount of ammonia, indicating that it also has high urease activity. Urea is difficult to remove from the extract, and the remaining urea may disturb the effective conversion of arginine to ornithine. In contrast, ammonia can easily be removed by lyophilisation. Therefore, we chose H. marmoreus as the source of arginase for the conversion of arginine in the garlic extracts. 
The ACSO content in Ge and GBe was 4.5 and $7.3 \mu \mathrm{mol} / \mathrm{g}$-extract, respectively, while that in HGe and HGBe was 81.8 and $65.5 \mu \mathrm{mol} / \mathrm{g}$-extract, respectively. This result indicates that C-S lyase was effectively inactivated by heating garlic at $80{ }^{\circ} \mathrm{C}$ for $30 \mathrm{~min}$. The arginine content in Ge and HGe was 183.1 and $181.4 \mu \mathrm{mol} / \mathrm{g}$-extract, respectively, while the ornithine content in GBe and HGBe was 127.9 and $149.1 \mu \mathrm{mol} / \mathrm{g}$-extract, respectively. Therefore, approximately $70-80 \%$ of arginine is converted to ornithine by arginase in $H$. marmoreus.

As $10 \%$ of $\mathrm{Ge}, \mathrm{GBe}, \mathrm{HGe}$, and $\mathrm{HGBe}$ in $1 \mathrm{~mL}$ of distilled water was orally administered to rats, the dose of ACSO in the Ge, GBe, HGe, HGBe groups was 0.45, 0.73, 8.18, and $6.55 \mu \mathrm{mol} /$ day (Table 2), respectively, which was approximately $1 / 100$ to $1 / 10$ the dose of synthesised ACSO in the former experiment. In contrast, the dose of arginine in the Ge and HGe groups was 18.31 and $18.14 \mu \mathrm{mol}$, respectively, while that of ornithine in the GBe and HGBe groups was 12.79 and 14.91, respectively. Although the doses of arginine and ornithine in these groups were approximately $1 / 3$ the dose in the former experiment, the hepatic injury was significantly suppressed in all the groups (Ge, GBe, HGe, and HGBe groups). Therefore, even less than $20 \mu \mathrm{mol}$ of arginine and ornithine would be effective to exert their function in the presence of ACSO and some other compounds in the extract. As volatile sulphides such as DADS and other amino acids that suppress $\mathrm{CCl}_{4}$-induced hepatic injury could be negligible in all garlic extracts, ACSO, arginine, and ornithine would be the major factors responsible for this effect.

Table 2. Daily dose of ACSO, arginine, and ornithine in the animal experiments.

\begin{tabular}{cccc}
\hline Group & ACSO $(\mu \mathrm{mol})$ & Arginine $(\mu \mathrm{mol})$ & Ornithine $(\mu \mathrm{mol})$ \\
\hline Ge & 0.45 & 18.31 & 0.15 \\
GBe & 0.73 & 0.23 & 12.79 \\
HGe & 8.18 & 18.14 & 0.23 \\
HGBe & 6.55 & 0.06 & 14.91 \\
\hline
\end{tabular}

The taste of bioactive compounds is important for their use in functional foods. ACSO is tasteless, odourless, and reportedly enhances the taste of food [24]. Arginine has a bitter taste [46], whereas ornithine has a flat or sweet taste [47,48]. Moreover, ornithine masks the bitter taste better than arginine [48]. Although precise mechanisms should be clarified for the hepatoprotective effect by multi-components, the conversion of arginine to ornithine in garlic using mushroom extract could offer a food material that protects the liver and improves the palatability of food.

\section{Conclusions}

The co-administration of ornithine/arginine with ACSO suppressed $\mathrm{CCl}_{4}$-induced hepatic injury in rats more potently than a single administration of ACSO, probably owing to synergistic effects. The extract with high contents of ACSO and ornithine was prepared from garlic and H. marmoreus (buna-shimeji), which converted arginine in garlic to ornithine by arginase. All garlic extracts with low and high ACSO contents and with arginine or ornithine alleviated $\mathrm{CCl}_{4}$-induced hepatic injury in rats. Considering that ornithine has a flat or sweet taste and masks bitter taste, the extract with high contents of ACSO and ornithine can be used as an antioxidant food material to protect against hepatic injury.

Supplementary Materials: The following are available online at https:/ / www.mdpi.com/article/10 .3390 / foods10071491/s1, Table S1: Contents of amino acids in garlic and bunashimeji extracts.

Author Contributions: Conceptualization, T.S. and H.K.; methodology, Y.H., T.S., and H.K.; validation, Y.Y., Y.H., T.S., and H.K.; formal analysis, Y.Y., Y.H., T.S., and H.K.; investigation, Y.Y., Y.H., and T.S.; data curation, Y.H. and T.S.; writing-original draft preparation, Y.Y.; writing-review and editing, H.K.; visualization, Y.Y., Y.H., and H.K.; supervision, H.K.; project administration, H.K. All authors have read and agreed to the published version of the manuscript. 
Funding: This research received no external funding.

Institutional Review Board Statement: The study was conducted according to the guidelines of the Declaration of Helsinki and approved by the Animal Experimental Committee of the College of Bioresource Sciences, Nihon University (approval code: AP13B010).

Informed Consent Statement: Not applicable.

Data Availability Statement: The data presented in this study are available on request from the corresponding author. The data are not publicly available due to their large volume and little interest.

Conflicts of Interest: The authors declare that they have no conflict of interest.

\section{References}

1. Markesbery, W.R. Oxidative stress hypothesis in alzheimer's disease. Free Radic. Biol. Med. 1997, 23, 134-147. [CrossRef]

2. Madamanchi, N.R.; Vendrov, A.; Runge, M.S. Oxidative stress and vascular disease. Arterioscler. Thromb. Vasc. Biol. 2005, 25, 29-38. [CrossRef] [PubMed]

3. Reuter, S.; Gupta, S.C.; Chaturvedi, M.M.; Aggarwal, B.B. Oxidative stress, inflammation, and cancer: How are they linked? Free Radic. Biol. Med. 2010, 49, 1603-1616. [CrossRef]

4. Bjelakovic, G.; Nikolova, D.; Gluud, L.L.; Simonetti, R.G.; Gluud, C. Antioxidant supplements for prevention of mortality in healthy participants and patients with various diseases. Cochrane Database Syst. Rev. 2012. [CrossRef] [PubMed]

5. Zhang, Y.-J.; Gan, R.-Y.; Li, S.; Zhou, Y.; Li, A.-N.; Xu, D.-P.; Li, H.-B. Antioxidant phytochemicals for the prevention and treatment of chronic diseases. Molecules 2015, 20, 21138-21156. [CrossRef] [PubMed]

6. Liu, Z.; Ren, Z.; Zhang, J.; Chuang, C.-C.; Kandaswamy, E.; Zhou, T.; Zuo, L. Role of ROS and nutritional antioxidants in human diseases. Front. Physiol. 2018, 9, 477. [CrossRef]

7. Forester, S.C.; Lambert, J.D. The role of antioxidant versus pro-oxidant effects of green tea polyphenols in cancer prevention. Mol. Nutr. Food Res. 2011, 55, 844-854. [CrossRef] [PubMed]

8. Jiang, Q. Natural forms of vitamin E: Metabolism, antioxidant, and anti-inflammatory activities and their role in disease prevention and therapy. Free Radic. Biol. Med. 2014, 72, 76-90. [CrossRef] [PubMed]

9. Lobo, V.; Patil, A.; Phatak, A.; Chandra, N. Free radicals, antioxidants and functional foods: Impact on human health. Pharmacogn. Rev. 2010, 4, 118. [CrossRef]

10. Shahidi, F. Functional foods: Their role in health promotion and disease prevention. J. Food Sci. 2006, 69, R146-R149. [CrossRef]

11. Moosavian, S.P.; Arab, A.; Paknahad, Z.; Moradi, S. The effects of garlic supplementation on oxidative stress markers: A systematic review and meta-analysis of randomized controlled trials. Complement. Ther. Med. 2020, 50, 102385. [CrossRef] [PubMed]

12. Lawson, L.D. Garlic: A review of its medicinal effects and indicated active compounds. In ACS Symposium Series; American Chemical Society: Washington, DC, USA, 1998; pp. 176-209.

13. Khanum, F.; Anilakumar, K.R.; Viswanathan, K.R. Anticarcinogenic properties of garlic: A review. Crit. Rev. Food Sci. Nutr. 2004, 44, 479-488. [CrossRef]

14. Gebreyohannes, G.; Gebreyohannes, M. Medicinal values of garlic: A review. Int. J. Med. Sci. 2013, 5, 401-408.

15. Ariga, T.; Seki, T. Antithrombotic and anticancer effects of garlic-derived sulfur compounds: A review. BioFactors 2006, 26, 93-103. [CrossRef] [PubMed]

16. Seki, T.; Hosono, T.; Hosono-Fukao, T.; Inada, K.; Tanaka, R.; Ogihara, J.; Ariga, T. Anticancer effects of diallyl trisulfide derived from garlic. Asia Pac. J. Clin. Nutr. 2008, 1, 249-252.

17. Yi, L.; Su, Q. Molecular mechanisms for the anti-cancer effects of diallyl disulfide. Food Chem. Toxicol. 2013, 57, 362-370. [CrossRef]

18. Zeng, T.; Zhang, C.-L.; Song, F.-Y.; Zhao, X.-L.; Yu, L.-H.; Zhu, Z.-P.; Xie, K.-Q. The activation of HO-1/Nrf-2 contributes to the protective effects of diallyl disulfide (DADS) against ethanol-induced oxidative stress. Biochim. Biophys. Acta 2013, 1830, 4848-4859. [CrossRef] [PubMed]

19. Liu, K.-L.; Chen, H.-W.; Wang, R.-Y.; Lei, Y.-P.; Sheen, L.-Y.; Lii, C.-K. DATS reduces LPS-induced INOS expression, NO production, oxidative stress, and NF-KB activation in RAW 264.7 macrophages. J. Agric. Food Chem. 2006, 54, 3472-3478. [CrossRef]

20. Zeng, T.; Zhang, C.-L.; Zhu, Z.-P.; Yu, L.-H.; Zhao, X.-L.; Xie, K.-Q. Diallyl trisulfide (DATS) effectively attenuated oxidative stress-mediated liver injury and hepatic mitochondrial dysfunction in acute ethanol-exposed mice. Toxicology 2008, $252,86-91$. [CrossRef]

21. Leung, W.-S.; Kuo, W.-W.; Ju, D.-T.; Wang, T.-D.; Shao-Tsu Chen, W.; Ho, T.-J.; Lin, Y.M.; Mahalakshmi, B.; Lin, J.-Y.; Huang, C.-Y. Protective effects of diallyl trisulfide (DATS) against doxorubicin-induced inflammation and oxidative stress in the brain of rats. Free Radic. Biol. Med. 2020, 160, 141-148. [CrossRef]

22. Yu, L.; Li, S.; Tang, X.; Li, Z.; Zhang, J.; Xue, X.; Han, J.; Liu, Y.; Zhang, Y.; Zhang, Y.; et al. Diallyl trisulfide ameliorates myocardial ischemia-reperfusion injury by reducing oxidative stress and endoplasmic reticulum stress-mediated apoptosis in type 1 diabetic rats: Role of SIRT1 activation. Apoptosis 2017, 22, 942-954. [CrossRef]

23. Yamaguchi, Y.; Kumagai, H. Characteristics, biosynthesis, decomposition, metabolism and functions of the garlic odour precursor, S-allyl-L-cysteine sulfoxide (review). Exp. Ther. Med. 2019, 19, 1528-1535. [CrossRef] 
24. Ueda, Y.; Sakaguchi, M.; Hirayama, K.; Miyajima, R.; Kimizuka, A. Characteristic flavor constituents in water extract of garlic. Agric. Biol. Chem. 1990, 54, 163-169.

25. Yamaguchi, Y.; Honma, R.; Yazaki, T.; Shibuya, T.; Sakaguchi, T.; Uto-Kondo, H.; Kumagai, H. Sulfuric odor precursor S-allyl-Lcysteine sulfoxide in garlic induces detoxifying enzymes and prevents hepatic injury. Antioxidants 2019, 8, 385. [CrossRef]

26. Najmi, A.; Pillai, K.; Pal, S.; Akhtar, M.; Aqil, M.; Sharma, M. Effect of L-ornithine L-aspartate against thioacetamide-induced hepatic damage in rats. Indian J. Pharmacol. 2010, 42, 384. [CrossRef] [PubMed]

27. Wagemaker, M.J.M.; Welboren, W.; van der Drift, C.; Jetten, M.S.M.; Van Griensven, L.J.L.D.; Op den Camp, H.J.M. The ornithine cycle enzyme arginase from Agaricus bisporus and its role in urea accumulation in fruit bodies. Biochim. Biophys. Acta 2005, 1681, 107-115. [CrossRef]

28. Jayakumar, T.; Ramesh, E.; Geraldine, P. Antioxidant activity of the oyster mushroom, pleurotus ostreatus, on CCl4-induced liver injury in rats. Food Chem. Toxicol. 2006, 44, 1989-1996. [CrossRef] [PubMed]

29. Huo, H.Z.; Wang, B.; Liang, Y.K.; Bao, Y.Y.; Gu, Y. Hepatoprotective and antioxidant effects of licorice extract against $\mathrm{CCl}_{4}$-induced oxidative damage in rats. Int. J. Mol. Sci. 2011, 12, 6529-6543. [CrossRef] [PubMed]

30. Cheng, N.; Ren, N.; Gao, H.; Lei, X.; Zheng, J.; Cao, W. Antioxidant and hepatoprotective effects of schisandra chinensis pollen extract on $\mathrm{CCl}_{4}$-induced acute liver damage in mice. Food Chem. Toxicol. 2013, 55, 234-240. [CrossRef]

31. Pan, Y.; Long, X.; Yi, R.; Zhao, X. Polyphenols in liubao tea can prevent $\mathrm{CCl}_{4}$-induced hepatic damage in mice through its antioxidant capacities. Nutrients 2018, 10, 1280. [CrossRef]

32. Hakamata, W.; Koyama, R.; Tanida, M.; Haga, T.; Hirano, T.; Akao, M.; Kumagai, H.; Nishio, T. A simple synthesis of alliin and allo-alliin: X-ray diffraction analysis and determination of their absolute configurations. J. Agric. Food Chem. 2015, 63, 10778-10784. [CrossRef] [PubMed]

33. Haber, D.; Siess, M.-H.; De Waziers, I.; Beaune, P.; Suschetet, M. Modification of hepatic drug-metabolizing enzymes in rat fed naturally occurring allyl sulphides. Xenobiotica 1994, 24, 169-182. [CrossRef] [PubMed]

34. Chang, T.K.H.; Crespi, C.L.; Waxman, D.J. Spectrophotometric analysis of human CYP2E1-Catalyzed $p$-nitrophenol hydroxylation. Methods Mol. Biol. 2006, 320, 127-132. [CrossRef]

35. De Toranzo, E.G.D.; De Ferreyra, E.C.; De Fenos, O.M.; Castro, J.A. Prevention of carbon tetrachloride-induced liver necrosis by several amino acids. Br. J. Exp. Pathol. 1983, 64, 166-171.

36. Akao, M.; Shibuya, T.; Shimada, S.; Sakurai, H.; Kumagai, H. In vivo production of bioactive compounds from S-allyl-L-cysteine sulfoxide, garlic odor precursor, that inhibit platelet aggregation. J. Clin. Biochem. Nutr. Suppl. 2008, 43, 1-3.

37. Uto-Kondo, H.; Hase, A.; Yamaguchi, Y.; Sakurai, A.; Akao, M.; Saito, T.; Kumagai, H. S-Allyl-L-cysteine sulfoxide, a garlic odor precursor, suppresses elevation in blood ethanol concentration by accelerating ethanol metabolism and preventing ethanol absorption from gut. Biosci. Biotechnol. Biochem. 2018, 82, 724-731. [CrossRef]

38. Raucy, J.L.; Kraner, J.C.; Lasker, J.M. Bioactivation of halogenated hydrocarbons by cytochrome P4502E1. Crit. Rev. Toxicol. 1993, 23, 1-20. [CrossRef]

39. Weber, L.W.D.; Boll, M.; Stampfl, A. Hepatotoxicity and mechanism of action of haloalkanes: Carbon tetrachloride as a toxicological model. Crit. Rev. Toxicol. 2003, 33, 105-136. [CrossRef]

40. Recknagel, R.O. A New direction in the study of carbon tetrachloride hepatotoxicity. Life Sci. 1983, 33, 401-408. [CrossRef]

41. Slater, T.F. Free-radical mechanisms in tissue injury. Biochem. J. 1984, 222, 1-15. [CrossRef] [PubMed]

42. Ramaiah, S.K. A Toxicologist guide to the diagnostic interpretation of hepatic biochemical parameters. Food Chem. Toxicol. 2007, 45, 1551-1557. [CrossRef] [PubMed]

43. Liang, M.; Wang, Z.; Li, H.; Cai, L.; Pan, J.; He, H.; Wu, Q.; Tang, Y.; Ma, J.; Yang, L. L-Arginine induces antioxidant response to prevent oxidative stress via stimulation of glutathione synthesis and activation of Nrf2 pathway. Food Chem. Toxicol. 2018, 115, 315-328. [CrossRef]

44. Gebhardt, R.; Beckers, G.; Guanitz, F.; Haupt, W.; Jonitza, D.; Klein, S. Treatment of cirrhotic rats with L-ornithine-L-aspartate enhances urea synthesis and lowers serum ammonia levels. J. Pharmacol. Exp. Ther. 1997, 283, 1-6.

45. Cynober, L.; Boucher, J.L.; Vasson, M.-P. Arginine metabolism in mammals. J. Nutr. Biochem. 1995, 6, 402-413. [CrossRef]

46. Schiffman, S.S.; Sennewald, K.; Gagnon, J. Comparison of taste qualities and thresholds of D and L-amino acids. Physiol. Behav. 1981, 27, 51-59. [CrossRef]

47. Asao, M.; Iwamura, H.; Akamatsu, M.; Fujita, T. Quantitative structure-activity relationships of the bitter thresholds of amino acids, peptides, and their derivatives. J. Med. Chem. 1987, 30, 1873-1879. [CrossRef] [PubMed]

48. Tokuyama, E.; Shibasaki, T.; Kawabe, H.; Mukai, J.; Okada, S.; Uchida, T. Bitterness suppression of BCAA solutions by L-ornithine. Chem. Pharm. Bull. 2006, 54, 1288-1292. [CrossRef] 